\title{
VALORACIÓN DE OBJETOS EDUCATIVOS ENRIQUECIDOS CON REALIDAD AUMENTADA: UNA EXPERIENCIA CON ALUMNADO DE MÁSTER UNIVERSITARIO
}

\section{VALUATION OF THE EDUCATIONAL OBJECTS WITH AUGMENTED REALITY: EXPERIENCE WITH POSGRADUATES STUDENTS}

\author{
Dra. Urtza Garay Ruiz \\ urtza.garay@ehu.eus \\ Dr. Eneko Tejada Garitano \\ eneko.tejada@ehu.eus \\ Dra. Inmaculada Maiz Olazabalaga \\ inmaculada.maiz@ehu.eus
}

\begin{abstract}
Universidad del País Vasco/Euskal Herriko Unibertsitatea
E.U. de Magisterio de Bilbao. Departamento de Psicología evolutiva y de la educación.

Barrio Sarriena s/n 48940 Leioa. Bizkaia (España)
\end{abstract}

\begin{abstract}
La realidad aumentada se ha convertido en una tecnología emergente y es necesario analizar su impacto en la educación. En este artículo se analiza la valoración del alumnado de postgrado sobre objetos educativos enriquecidos con RA. Para ello se utiliza una encuesta tipo Likert que indaga sobre las características de este tipo de materiales: aspectos técnicos y estéticos, facilidad de navegación y desplazamiento por el entorno y guía/tutorial. El estudio pone de manifiesto que la característica que mejor se valora de RA es la facilidad de navegación: objetos intuitivos, rápidos en su respuesta y sencillos de utilizar.

Palabras clave: Realidad Aumentada, investigación, educación.
\end{abstract}

Augmented reality has become an emerging technology, so there is a need to investigate its impact on education. In this article we research the assessment of graduate students on educational objects with RA. For this we use a Likert survey that explores the characteristics of this type of materials: technical and aesthetic aspects, ease of navigation and scrolling through the environment and tutorial guide. The study results indicate that the characteristic that students best value is that the object with RA easy to use, fast and intuitive.

Keywords: Augmented Reality, research, education

$$
-19-
$$

Píxel-Bit. Revista de Medios y Educación. No 50 Enero 2017. ISSN: 1133-8482. e-ISSN: 2171-7966. doi: http://dx.doi.org/10.12795/pixelbit.2017.i50.01 


\section{Introducción.}

La introducción de dispositivos móviles en las aulas conlleva un cambio en la forma de aprender y también de enseñar (Castaño \& Cabero, 2013). El conocido m-learning o el aprendizaje en movilidad ha supuesto que diferentes tecnologías emergentes, entre ellas, la Realidad Aumentada encuentren su lugar en el contexto de la educación (UNESCO, 2012). En esta misma línea en los últimos años se está consolidando la idea de que la Realidad Aumentada se constituye como un elemento base de la educación en un futuro cercano (Cabero, García \& Barroso, 2016; Durall, Gros, Maina, Johnson \& Adams, 2012; Johnson et. al, 2013).

La Realidad Aumentada no es una tecnología nueva, tiene un desarrollo tecnológico amplio a pesar de que no se ha explotado lo suficiente todavía en el ámbito educativo. Actualmente, desde el punto de vista tecnológico se entiende por Realidad Aumentada enriquecer la información física con información digital en tiempo real mediante dispositivos tecnológicos que permiten descifrarla (Basogain, Olabe, Espinosa, Roueche \& Olabe, 2007; García et al. 2010; Muñoz, 2013). Por lo que la virtud de este tipo de tecnología es que permite al usuario interactuar con los objetos de aprendizaje (Cabero \& Barroso, 2015).

Wu, Wen y Yi (2013) añaden más beneficios y señalan que contribuye no solo a reducir la distancia que existe entre el aprendizaje formal e informal, sino a habilitar un aprendizaje ubicuo, situado y enriquecido, facilitando la comprensión de conceptos complejos. Estudios previos han demostrado que la realidad aumentada puede mejorar el rendimiento académico del alumnado (Chiu, DeJaegher \& Chao, 2015) y ayudar al desarrollo de las competencias y habilidades de manera más eficaz que otras tecnologías (ElSayed, Zayed \& Sharawy, 2011). Por otra parte también se observa un aumento en la motivación de los estudiantes universitarios (Akçayir, Akçayir, Pektas \& Ocak, 2016).

De este modo, a pesar de que existen multitud de experiencias de utilización de materiales educativos enriquecidos con Realidad Aumentada (Prendes, 2015) en casi todos los niveles educativos formales (Bongiovani, 2013; De Pedro \& Méndez, 2012; Gutierrez, 2014; Pei-Hsun y Ming-Kuan, 2013), todavía se ve la necesidad de seguir investigando y analizando las posibilidades educativas que presenta la utilización de la realidad aumentada (Cabero y García, 2016).

Este trabajo pretende contribuir en esta línea de investigación. Así se busca analizar la satisfacción del alumnado universitario de postgrado en relación a objetos de aprendizaje enriquecidos con RA. En resumen, ver qué aspectos valoran mejor los alumnos cuando viven una experiencia de aprendizaje basada en realidad aumentada, y así buscar vías de inclusión de la tecnología conocida como realidad aumentada en las aulas universitarias actuales y futuras.

\section{Metodología y muestra.}

El objetivo de la investigación es conocer la valoración del alumnado sobre las características de los objetos enriquecidos con Realidad Aumentada susceptibles de facilitar el aprendizaje. Para ello se formularon las siguientes preguntas de investigación:

1. ¿Qué característica de los objetos educativos con Realidad Aumentada es la mejor valorada por los estudiantes?

2. ¿Influyen las características propias del alumnado (edad y género) en la valoración de los objetos educativos basados RA?

$$
-20-
$$


3. ¿Los estudios de procedencia del alumnado influyen de forma significativa a la hora de valorar objetos educativos enriquecidos con RA?

La experiencia se realizó en Santo Domingo (República Dominicana) dentro del Máster Universitario Tecnología, Aprendizaje y Educación que imparte la Universidad del País Vasco (UPV-EHU). El proceso consistía en utilizar, analizar y valorar nueve objetos de aprendizaje basados en el uso de la Realidad Aumentada. A continuación se describen características de cada uno de ellos (ver Tabla 1).

Debemos señalar que para interaccionar con el programa se debe descargar de Google Play, en el dispositivo móvil, la app correspondiente a cada objeto de RA. Las apps también están en versión Windows, sin embargo, al estar pensadas para utilizarse con una webcam, tienen limitaciones en las interacciones que requieren de una pantalla táctil. También es necesario tener conexión a Internet ya que algunos de los recursos que se presentan están en soporte vídeo. Además tal y como se ha podido observar en la descripción (ver Tabla 1) hay objetos que necesitan tener la función de geolocalización activada.

Para la evaluación de los objetos se utilizó una encuesta basada en una escala Likert de 6 opciones (MP, muy positivo; P, positivo; $\mathrm{R}+$, regular-positivo,; R-, regular-negativo; $\mathrm{N}$, negativo y $\mathrm{MN}$, muy negativo) producida para el proyecto RAFODIUN (Universidad de Sevilla) y que constaba de tres bloques de ítems que constituyen las variables dependientes del estudio. Los bloques son las siguientes: «Aspectos técnicos y estéticos», «Facilidad de navegación y desplazamiento por el entorno» y «Guía/ Tutorial del programa».
Cada uno de los bloques estaba constituido por un número determinado de ítems que englobaban las características básicas del objeto de aprendizaje enriquecido con Realidad Aumentada. En relación a los aspectos técnicos y estéticos se especificaron cuatro ítems que hacían referencia al funcionamiento, la estética y la presentación de la información en la pantalla.

En lo que se refiere a la «Facilidad de navegación y desplazamiento por el entorno» mediante seis ítems se preguntó sobre la percepción en relación a la facilidad de manejo y facilidad de comprensión del funcionamiento técnico del objeto, además de su diseño, accesibilidad, flexibilidad y disfrute.

Finalmente en el último bloque conformado por dos ítems de contraste, y que analizaba la guía o tutorial del programa que se entregaba al alumnado para la comprensión y utilización del objeto educativo enriquecido con RA, se preguntaba sobre la información que se daba, su eficacia y facilidad de comprensión.

La muestra la conformaron 32 alumnos, 17 hombres y 15 mujeres, de edades comprendidas entre los 24 años hasta mayores de 50 y que pertenecían a diferentes áreas del conocimiento (Arte y Humanidades, Ciencias, Ciencias Sociales y Jurídicas e Ingeniería y Arquitectura). Esto es, habían realizado sus estudios universitarios en diferentes ramas de conocimiento y en la actualidad estaban cursando el Máster Universitario Tecnología, Aprendizaje y Educación de la Universidad del País Vasco (UPV-EHU). El análisis de los datos se realizó con el programa SPSS versión 22.

$$
-21-
$$

Píxel-Bit. Revista de Medios y Educación. No 50 Enero 2017. ISSN: 1133-8482. PIXEL e-ISSN: 2171-7966. doi: http://dx.doi.org/10.12795/pixelbit.2017.i50.01 


\begin{tabular}{|c|c|c|}
\hline $\begin{array}{l}\text { NÚMERO } \\
\text { DE } \\
\text { OBJETO }\end{array}$ & NOMBRE & CARACTERÍSTICAS TÉCNICAS Y ESTÉTICAS \\
\hline 1 & $\begin{array}{l}\text { Anatomía humana: cervical } \\
\text { (App: CERVICAL2) }\end{array}$ & $\begin{array}{l}\text { La RA se lanza a través de la imagen de la figura ósea de un cuerpo } \\
\text { humano. En la parte superior derecha se señala el tamaño del } \\
\text { encuadre que debe tener la cámara sobre el objeto. En la parte } \\
\text { superior izquierda se muestra la zona anatómica que va a ser } \\
\text { aumentada. } \\
\text { La lectura con la app ofrece dos pestañas que corresponden a dos } \\
\text { perspectivas del mismo hueso y que ofrecen las mismas } \\
\text { características: un vídeo explicativo de imágenes y audio (duración } \\
9 \text { min } 45 \text { s.) y una imagen en } 3 \text { D manipulable en tamaño. }\end{array}$ \\
\hline 2 & $\begin{array}{l}\text { Anatomía humana: } \\
\text { coxofemoral }\end{array}$ & $\begin{array}{l}\text { La RA se lanza a través de la imagen de la figura ósea de un cuerpo } \\
\text { humano. En la parte superior derecha se señala el tamaño del } \\
\text { encuadre que debe tener la cámara sobre el objeto. En la parte } \\
\text { central izquierda se muestra la zona anatómica que va a ser } \\
\text { aumentada. } \\
\text { Con la lectura de la app emerge el hueso correspondiente en 3D que } \\
\text { rota y que se puede detener cuando se desee. Al tocar el hueso se } \\
\text { dan dos opciones: } \\
\text {-ver huesos de alrededor } \\
\text {-ver vídeo explicativo basado en imágenes y audio (duración } 21 \text { min } \\
18 \text { s.) y poder manipular (cambiar de tamaño y alejar/acercar) el } \\
\text { hueso coxofemoral. }\end{array}$ \\
\hline 3 & $\begin{array}{l}\text { Histología humana: hígado } \\
\text { (App: ARUSLIVER) }\end{array}$ & $\begin{array}{l}\text { La RA se lanza a través de la imagen de la figura de un hígado } \\
\text { sobreimpresionado en la parte central de un cuerpo humano. } \\
\text { El lanzador es muy rápido, el objeto RA emerge de inmediato. } \\
\text { Con la app emerge un esquema donde se ve la figura del hígado en } \\
\text { 3D (manipulable en tamaño, distancia y dirección de giro) y tres } \\
\text { apartados que corresponden a la temática. Tocando cada apartado } \\
\text { aparece un podcast muy breve (duración } 2 \text { min aprox.) } \\
\text { acompañado de una imagen plana en 3D con la opción de elegir la } \\
\text { vista que a uno le interese. }\end{array}$ \\
\hline 4 & $\begin{array}{l}\text { Anatomía humana: hombro } \\
\text { (App: SHOULDER2) }\end{array}$ & $\begin{array}{l}\text { La RA se lanza a través de la imagen de la figura ósea de un cuerpo } \\
\text { humano. En la parte superior derecha se señala el tamaño del } \\
\text { encuadre que debe tener la cámara sobre el objeto. } \\
\text { En la parte superior izquierda se muestra la zona anatómica que va } \\
\text { a ser aumentada. } \\
\text { Con la lectura de la app emerge el hueso correspondiente en 3D y } \\
\text { rota, se puede detener cuando se desee. Al tocar el hueso hay dos } \\
\text { opciones: } \\
\text {-ver los huesos de alrededor }\end{array}$ \\
\hline
\end{tabular}

Tabla 1: Características de los objetos educativos enriquecidos con RA. Los objetos se pueden encontrar en: http://intra.sav.us.es/proyectorafodiun/index.php/objetos-en-ra

$$
-22-
$$




\begin{tabular}{|c|c|c|}
\hline $\begin{array}{l}\text { NÚMERO } \\
\text { DE } \\
\text { OBJETO }\end{array}$ & NOMBRE & CARACTERÍSTICAS TÉCNICAS Y ESTÉTICAS \\
\hline 5 & $\begin{array}{l}\text { Histología Humana: } \\
\text { pulmón } \\
\text { (App: ARUSLUNG) }\end{array}$ & $\begin{array}{l}\text { La RA se lanza a través de la imagen de la figura de unos pulmones de color rojo dentro } \\
\text { de una caja torácica azul. } \\
\text { El lanzador es muy rápido, el objeto RA emerge de inmediato. } \\
\text { Con la app emerge un esquema donde se ve la figura de los pulmones en 3D manipulable } \\
\text { en tamaño, distancia y dirección de giro. Además tres apartados que corresponden a la } \\
\text { temática. Cada apartado nos lleva a un podcast muy breve (duración } 1,5 \text { min aprox.) } \\
\text { acompañado de una imagen plana en 3D con la opción de elegir la vista que a uno le } \\
\text { interese. }\end{array}$ \\
\hline 6 & $\begin{array}{l}\text { Anatomía humana: } \\
\text { tobillo } \\
\text { (App: ANKLE2) }\end{array}$ & $\begin{array}{l}\text { La RA se lanza a través de la imagen de la figura ósea de un cuerpo humano. En la parte } \\
\text { superior derecha se señala el tamaño del encuadre que debe tener la cámara sobre el } \\
\text { objeto. } \\
\text { En la parte inferior izquierda se muestra la zona anatómica que va a ser aumentada. } \\
\text { Con la lectura de la app emerge el hueso correspondiente en 3D y y rota, que se puede } \\
\text { detener cuando se desee. Al tocar el hueso hay dos opciones de elección: } \\
\text {-ver los huesos de alrededor } \\
\text {-ver el vídeo explicativo basado en imágenes y audio (duración } 12 \min 35 \mathrm{~s} \text {.) y manipular } \\
\text { (cambiar de tamaño y alejar/acercar) el hueso del tobillo. }\end{array}$ \\
\hline 7 & $\begin{array}{l}\text { Mapa Interactivo de los } \\
\text { Jardines del Monasterio } \\
\text { de la Cartuja de Sevilla } \\
\text { (App: CARTUJAPP) }\end{array}$ & $\begin{array}{l}\text { Secuencia de } 9 \text { imágenes de los Jardines del Monasterio de la Cartuja de Sevilla. } \\
\text { Exige tener la geolocalización activada en el dispositivo móvil que se utilice. } \\
\text { Consta de } 9 \text { lanzadores, que corresponden a las diferentes partes de la Cartuja. } \\
\text { Con la lectura de la app en cada lanzador emergen de la imagen tres pequeñas pestañas } \\
\text { que llevan una web informativa, a un podcast y a realizar la visita en imagen real 360, } \\
\text { pero con una visión limitada. }\end{array}$ \\
\hline 8 & $\begin{array}{l}\text { Diseño, producción y } \\
\text { evaluación de Tecnologías } \\
\text { de la Información y } \\
\text { Comunicación aplicadas a } \\
\text { la educación } \\
\text { (App: RAFODIUN) }\end{array}$ & $\begin{array}{l}\text { La RA se lanza a través de un esquema que resume el contenido de aprendizaje: la } \\
\text { creación de materiales TIC para educación. } \\
\text { Es un lanzador muy completo, que tiene la opción de saltar aquello que se ha visto en } \\
\text { otra ocasión. Ofrece la oportunidad de verlo en diferentes tiempos, ya que lanza mucha } \\
\text { información. } \\
\text { En la lectura con la app lo primero que emerge es un vídeo transparente de introducción } \\
\text { que explica el proceso de diseño, producción y evaluación de las TIC aplicadas a la } \\
\text { enseñanza. } \\
\text { Los apartados del lanzador se constituyen con las fases de creación de material TIC. Así } \\
\text { se va explicando cada fase utilizando vídeos con la imagen del profesor hablando que } \\
\text { rondan de } 2 \text { a } 5 \text { minutos, videos transparentes, textos de lectura con mapas conceptuales, } \\
\text { objetos } 3 \text { D rotativos, y actividades de evaluación. } \\
\text { El lanzador es muy rápido, el objeto RA emerge de inmediato. }\end{array}$ \\
\hline 9 & $\begin{array}{l}\text { Formas de utilizar el } \\
\text { vídeo en la enseñanza. } \\
(\mathrm{App}: \mathrm{VIEA} S \mathrm{SAV})\end{array}$ & $\begin{array}{l}\text { La RA se lanza a través de una imagen perteneciente al video que se lanza. } \\
\text { La lectura con la app emerge un video ( } 12 \text { min aprox.) donde el profesor explica el tema } \\
\text { que se va a tratar. } \\
\text { El tiempo de descarga de la app para su visualización es superior al de los demás objetos. } \\
\text { Aunque el lanzador es muy rápido, el objeto RA emerge de inmediato. }\end{array}$ \\
\hline
\end{tabular}

Tabla 1: Características de los objetos educativos enriquecidos con RA. Los objetos se pueden encontrar en: http://intra.sav.us.es/proyectorafodiun/index.php/objetos-en-ra

\section{(continuación)}

$$
-23-
$$

Píxel-Bit. Revista de Medios y Educación. No 50 Enero 2017. ISSN: 1133-8482. PIXEL e-ISSN: 2171-7966. doi: http://dx.doi.org/10.12795/pixelbit.2017.i50.01 


\section{Resultados.}

A continuación presentamos lo resultados de la investigación siguiendo las preguntas de investigación que se plantearon.

En relación a la primera pregunta de investigación (¿Qué característica de los objetos educativos con Realidad Aumentada es la mejor valorada por los estudiantes?) encontramos que entre las tres características que conformaban las variables independientes de este estudio, la mejor valorada por los estudiantes ha sido la segunda: Facilidad de navegación y desplazamiento por el entorno. Las medias recibidas por este apartado en todos los objetos abarcan del 9,97 al 13, 52. Si realizamos una clasificación entre los objetos teniendo en cuenta su facilidad de navegación y desplazamiento vemos que el objeto mejor valorado ha sido el objeto 3. Después con una media de alrededor de 11 estarían el objeto 5 o pulmón (media 11,88), el 6 o tobillo (media 11,43), el 4 u hombro (media 11,03). En un tercer escalón se encuentran los objetos 1 cervical $(10,97), 2$ coxofemoral (10.96) y 8 Diseño de TICs $(10,37)$. Y entre los objetos que peores resultados han obtenido se encuentran el objeto 9 utilización de vídeos en la enseñanza $(9,97)$ y el objeto 7 Monasterio de la Cartuja (9.21).

En un segundo nivel de valoración se encuentra el apartado dedicado a los aspectos técnicos y estéticos. En todos los objetos es el segundo aspecto mejor valorado, con una diferencia media de cuatro puntos en relación a la facilidad de navegación.

Tal y como se observa en la Tabla 2 , donde mejor se valoran los aspectos técnicos y estéticos es en el objeto 9 (Formas de utilizar los vídeos en la enseñanza), que curiosamente es de los que peor se valoran en relación a la facilidad de navegación y desplazamiento por el entorno. En cambio el objeto peor valorado es el 7 (Mapa Interactivo de los Jardines del Monasterio de la Cartuja de Sevilla), que es también el que recibe peor valoración en relación a su facilidad de navegación y desplazamiento por el entorno.

El aspecto peor valorado y con bastante diferencia, una media de 8 puntos en relación con la Facilidad de navegación y desplazamiento por el entorno y de 3 puntos con el Aspectos técnicos y estéticos se encuentra la Guía/Tutorial del programa. Es el aspecto peor valorado en todos los objetos, destacando de nuevo el objeto 7 (Mapa

\begin{tabular}{|c|c|c|c|}
\hline OBJETO & \multicolumn{3}{|c|}{ VALORACIÓN (MEDIA) } \\
\hline & Aspectos técnicos & $\begin{array}{c}\text { Facilidad de } \\
\text { navegación }\end{array}$ & Guía/Tutorial \\
\hline 1 & 6,60 & 10,97 & 3,20 \\
\hline 2 & 6,67 & 10,96 & 3,58 \\
\hline 3 & 8,81 & $\mathbf{1 3 , 5 2}$ & $\mathbf{4 , 0 6}$ \\
\hline 4 & 7,71 & 11,03 & 3,65 \\
\hline 5 & 8,03 & 11,88 & $\mathbf{4 , 0 6}$ \\
\hline 6 & 7,29 & 11,43 & 3,42 \\
\hline 7 & $\mathbf{6 , 2 1}$ & $\mathbf{9 , 2 1}$ & $\mathbf{2 , 7 9}$ \\
\hline 8 & 6,31 & 10,37 & 2,93 \\
\hline 9 & $\mathbf{9 , 9 7}$ & 9,97 & 3,03 \\
\hline
\end{tabular}

Tabla 2: Medias de las valoraciones de los objetos educativos enriquecidos con $R A$.

$$
-24-
$$


interactivo de los Jardines del Monasterio de la Cartuja de Sevilla) como el peor valorado con una media de 2,79. En contraposición, los objetos mejor valorados en relación a este aspecto han sido dos: el 3 (Histología humana: hígado) y el 5 (Histología humana: pulmón) con una media en ambos casos de 4,06 . En este caso el objeto tres repite como mejor valorado tal y como ocurría cuando se valoraba la Facilidad de navegación y desplazamiento por el entorno.

En la segunda pregunta (¿Influyen las características propias del alumnado (edad y género) en la valoración de los objetos educativos basados RA?) se preguntaba sobre la influencia de aspectos propios del alumnado como son la edad y el género en su valoración de la experiencia con objetivos educativos basados en RA. Después del análisis de las respuestas de los estudiantes se observa que, en general, ni el género ni la edad de los estudiantes influyen de forma significativa en la valoración de la experiencia. Aunque existe una excepción que se da en el objeto 1 (Anatomía humana: cervical). En este caso, mientras que el género no influye en la percepción de los estudiantes, la edad sí lo hace. Esta situación ocurre únicamente en el bloque que se refiere a los Aspectos técnicos y estéticos, donde encontramos significatividad entre los grupos de edad entre 20 y 29 años frente a los de 30-39 años (ver Tabla 3).

Para analizar con más profundidad estos resultados, después de la ANOVA se procesaron los datos mediante la prueba post hoc HSD Tukey donde se confirmaron los resultados. Se encontró diferencia significativa en los aspectos técnicos y estéticos entre el grupo de alumnos de 20-29 años y el de 30-39 años (Ver Tabla 4).

En todos los demás casos no existen diferencias significativas entre rangos de edad. A pesar de ello, tras un análisis de medias encontramos que en todos los objetos y en todas sus características los que mejor valoración presentan son los alumnos de entre 20 y 29 años, menos en dos ocasiones. En el objeto 7 (Mapa Interactivo de los Jardines del Monasterio de la Cartuja de Sevilla), en lo que se refiere a la guía, es el grupo de edad correspondiente a 40-49 años el que mejor lo valora. El apartado de Aspectos técnicos y estéticos del objeto número 8 (Diseño, producción y evaluación de Tecnologías de la Información y la

\section{ANOVA $^{\mathrm{a}}$}

\begin{tabular}{|c|c|c|c|c|c|c|}
\hline & & $\begin{array}{l}\text { Suma de } \\
\text { cuadrados }\end{array}$ & gl & $\begin{array}{c}\text { Media } \\
\text { cuadrática }\end{array}$ & $\mathrm{F}$ & Sig. \\
\hline \multirow[t]{3}{*}{ Aspectos Técnicos } & Entre grupos & 69,214 & 3 & 23,071 & 4,225 &, 015 \\
\hline & $\begin{array}{l}\text { Dentro de } \\
\text { grupos }\end{array}$ & 141,986 & 26 & 5,461 & & \\
\hline & Total & 211,200 & 29 & & & \\
\hline \multirow{3}{*}{$\begin{array}{l}\text { Facilidad de } \\
\text { navegación }\end{array}$} & Entre grupos & 39,877 & 3 & 13,292 & ,886 & ,462 \\
\hline & $\begin{array}{l}\text { Dentro de } \\
\text { grupos }\end{array}$ & 375,089 & 25 & 15,004 & & \\
\hline & Total & 414,966 & 28 & & & \\
\hline \multirow[t]{3}{*}{ Guía } & Entre grupos & 11,142 & 3 & 3,714 & ,969 & ,422 \\
\hline & $\begin{array}{l}\text { Dentro de } \\
\text { grupos }\end{array}$ & 99,658 & 26 & 3,833 & & \\
\hline & Total & 110,800 & 29 & & & \\
\hline
\end{tabular}

$-25-$

Píxel-Bit. Revista de Medios y Educación. No 50 Enero 2017. ISSN: 1133-8482. PIXEL e-ISSN: 2171-7966. doi: http://dx.doi.org/10.12795/pixelbit.2017.i50.01 


\begin{tabular}{|c|c|c|c|c|c|c|c|}
\hline \multirow[b]{2}{*}{$\begin{array}{l}\text { Variable } \\
\text { dependiente }\end{array}$} & \multirow[b]{2}{*}{ (I) edad } & \multirow[b]{2}{*}{ (J) edad } & \multirow{2}{*}{$\begin{array}{c}\text { Diferencia } \\
\text { de medias } \\
(\mathrm{I}-\mathrm{J})\end{array}$} & \multirow[b]{2}{*}{ Error estándar } & \multirow[b]{2}{*}{ Sig. } & \multicolumn{2}{|c|}{$\begin{array}{c}95 \% \text { de intervalo de } \\
\text { confianza }\end{array}$} \\
\hline & & & & & & $\begin{array}{l}\text { Límite } \\
\text { inferior }\end{array}$ & $\begin{array}{c}\text { Límite } \\
\text { superior }\end{array}$ \\
\hline \multirow{12}{*}{$\begin{array}{l}\text { Aspectos } \\
\text { Técnicos }\end{array}$} & \multirow[t]{3}{*}{2} & 3 & $3,427^{*}$ & 1,013 &, 012 &, 65 & 6,21 \\
\hline & & 4 & 3,289 & 1,303 &, 080 &,- 29 & 6,86 \\
\hline & & 5 & 2,556 & 1,558 & ,375 & $-1,72$ & 6,83 \\
\hline & \multirow[t]{3}{*}{3} & 2 & $-3,427^{*}$ & 1,013 &, 012 & $-6,21$ &,- 65 \\
\hline & & 4 &,- 138 & 1,230 & ,999 & $-3,51$ & 3,24 \\
\hline & & 5 &,- 872 & 1,497 & ,936 & $-4,98$ & 3,23 \\
\hline & \multirow[t]{3}{*}{4} & 2 & $-3,289$ & 1,303 &, 080 & $-6,86$ &, 29 \\
\hline & & 3 &, 138 & 1,230 & ,999 & $-3,24$ & 3,51 \\
\hline & & 5 &,- 733 & 1,707 &, 973 & $-5,42$ & 3,95 \\
\hline & \multirow[t]{3}{*}{5} & 2 & $-2,556$ & 1,558 &, 375 & $-6,83$ & 1,72 \\
\hline & & 3 & ,872 & 1,497 & ,936 & $-3,23$ & 4,98 \\
\hline & & 4 &, 733 & 1,707 & ,973 & $-3,95$ & 5,42 \\
\hline \multirow{12}{*}{$\begin{array}{l}\text { Facilidad de } \\
\text { navegación }\end{array}$} & \multirow[t]{3}{*}{2} & 3 & 2,611 & 1,708 & ,436 & $-2,09$ & 7,31 \\
\hline & & 4 & 2,044 & 2,161 & ,780 & $-3,90$ & 7,99 \\
\hline & & 5 &, 444 & 2,582 & ,998 & $-6,66$ & 7,55 \\
\hline & \multirow[t]{3}{*}{3} & 2 & $-2,611$ & 1,708 & ,436 & $-7,31$ & 2,09 \\
\hline & & 4 &,- 567 & 2,062 & ,993 & $-6,24$ & 5,10 \\
\hline & & 5 & $-2,167$ & 2,500 &, 822 & $-9,04$ & 4,71 \\
\hline & \multirow[t]{3}{*}{4} & 2 & $-2,044$ & 2,161 & ,780 & $-7,99$ & 3,90 \\
\hline & & 3 & ,567 & 2,062 & ,993 & $-5,10$ & 6,24 \\
\hline & & 5 & $-1,600$ & 2,829 &, 941 & $-9,38$ & 6,18 \\
\hline & \multirow[t]{3}{*}{5} & 2 &,- 444 & 2,582 & ,998 & $-7,55$ & 6,66 \\
\hline & & 3 & 2,167 & 2,500 & ,822 & $-4,71$ & 9,04 \\
\hline & & 4 & 1,600 & 2,829 & ,941 & $-6,18$ & 9,38 \\
\hline \multirow[t]{12}{*}{ Guía } & \multirow[t]{3}{*}{2} & 3 & 1,419 &, 849 & ,359 &,- 91 & 3,75 \\
\hline & & 4 & 1,111 & 1,092 &, 741 & $-1,88$ & 4,11 \\
\hline & & 5 & 1,111 & 1,305 & ,829 & $-2,47$ & 4,69 \\
\hline & \multirow[t]{3}{*}{3} & 2 & $-1,419$ &, 849 &, 359 & $-3,75$ & ,91 \\
\hline & & 4 &,- 308 & 1,030 & ,991 & $-3,13$ & 2,52 \\
\hline & & 5 &,- 308 & 1,254 & ,995 & $-3,75$ & 3,13 \\
\hline & \multirow[t]{3}{*}{4} & 2 & $-1,111$ & 1,092 & ,741 & $-4,11$ & 1,88 \\
\hline & & 3 & ,308 & 1,030 & ,991 & $-2,52$ & 3,13 \\
\hline & & 5 &, 000 & 1,430 & 1,000 & $-3,92$ & 3,92 \\
\hline & \multirow[t]{3}{*}{5} & 2 & $-1,111$ & 1,305 &, 829 & $-4,69$ & 2,47 \\
\hline & & 3 & ,308 & 1,254 & ,995 & $-3,13$ & 3,75 \\
\hline & & 4 &, 000 & 1,430 & 1,000 & $-3,92$ & 3,92 \\
\hline
\end{tabular}

*. La diferencia de medias es significativa en el nivel 0.05.

a. objeto $=1$

Tabla 4. Comparaciones múltiples HSD Tukey.

Comunicación aplicadas a la educación) es el grupo de mayores de 50 el que mejor lo valora. Debemos señalar que en estos dos casos se da otro fenómeno interesante: la diferencia entre medias. Así, el valor de esta diferencia entre el grupo que mejor y peor valora el objeto es el más bajo en ambos casos. En el objeto 7 (Mapa interactivo de los Jardines del Monasterio de la Cartuja de Sevilla) en relación a la Guía/Tutorial del programa, el

$$
-26-
$$


grupo de entre 40 y 49 años, que es el que mejor lo valora, obtiene una media de 3,20 y el grupo que peor lo valora, mayores de 50, una media de 2,50. Por lo tanto la diferencia entre ellos es mínima, un 0,7. Algo similar ocurre en lo referente a Aspectos técnicos y estéticos del objeto 8 (Diseño, producción y evaluación de Tecnologías de la Información y Comunicación aplicadas a la educación), donde son los mayores de 50 años los que mejor lo valoran con una media de 7,00, y los de 30 a 39 los que peor, con una media de 6,07. Así la diferencia entre ellos se puede considerar muy pequeña, ya que es de 0,93 .

En el lado opuesto, es decir, las valoraciones donde las diferencias de medias son las más altas se encuentran los objetos número 2 (Anatomía humana: coxofemoral) y 3 (Histología humana: hígado). Más concretamente, en los apartados de Aspectos técnicos y estéticos y Facilidad de navegación y desplazamiento por el entorno de estos objetos. En estos casos la diferencia entre el grupo de edad que mejor lo valora y el que peor lo valora superan los cinco puntos.

Entre ambos objetos, los resultados del objeto 2 (Anatomía humana: coxofemoral) son más homogéneos, ya que tanto en el bloque de Aspectos técnicos y estéticos como en el de Facilidad de navegación y desplazamiento por el entorno, el grupo de edad que mejor valora es el que engloba las edades entre 20 y 29 años, y el que lo valora peor el de mayores de 50 años. En relación a los Aspectos técnicos y estéticos la diferencia entre la media de los dos grupos es de 5,38 ( 20-29 años, 10,88 y mayores de $50,5,50$ ). En cambio en el aspecto Facilidad de navegación y desplazamiento por el entorno es donde se encuentra la mayor diferencia entre medias, un 5,71 (20-29 años, 15,88 y mayores de 50, $10,17)$.
Como ya hemos señalado, en el caso del objeto número dos hay más heterogeneidad entre las respuestas de las diferentes franjas de edad. Así, en la característica Aspectos técnicos y estéticos es el grupo entre 20 y 29 años el que mejor lo valora (11.00), pero hay dos grupos que coinciden en la valoración negativa, el de mayores de 50 años y el de 3039 años $(5,50)$. La diferencia entre los grupos es también superior a 5 , más concretamente 5,50. En el mismo objeto, en relación al apartado Facilidad de navegación y desplazamiento, se puede apreciar que sigue siendo el grupo de 20-29 el que valora de forma más positiva (14.00). En cambio los que presentan una valoración más negativa son los que están entre 40 y 49 años (9.00), siendo de 5 puntos la diferencia entre ambas medias.

Siguiendo esta misma línea, si nos adentramos en las diferencias entre género, tal y como hemos señalado, no existen diferencias significativas entre las valoraciones de los hombres y de las mujeres. Fijándonos en las medias se puede señalar que aunque de forma leve los hombres valoran mejor que las mujeres los objetos educativos enriquecidos con RA.

Así encontramos que los objetos 1 (cervical), 2 (coxofemoral), 8 (diseño y producción) y 9 (uso del vídeo en educación) se valoran mejor por los hombres que por las mujeres y los objetos número 3 (hígado) y 5 (pulmón) tienen mejor valoración de las mujeres. Finalmente hay objetos, como los objetos número 4 (hombro), 6 (tobillo) y 7 (mapa de la Cartuja), donde no se aprecian diferencias de valoraciones en ningunos de los aspectos que los caracterizan.

Las mayores diferencias de medias de valoración se encuentran en el apartado Aspectos técnicos y estéticos del objeto 1 (Anatomía humana: cervical), donde la media

$-27-$

Píxel-Bit. Revista de Medios y Educación. No 50 Enero 2017. ISSN: 1133-8482. PIXEL e-ISSN: 2171-7966. doi: http://dx.doi.org/10.12795/pixelbit.2017.i50.01 
de los hombres supera en 1,8 puntos a la de las mujeres (hombres 7,62 y mujeres 5,82). Lo mismo ocurre en el objeto 3 , pero en este caso son las mujeres las que mejor lo valoran. Esto es, en lo que se refiere al apartado Facilidad de navegación y desplazamiento por el entorno del objeto número tres, valoran de forma más positiva, con una diferencia de media de 1,34, ya que la media de la percepción de los hombres es 12,87 y la de las mujeres 14,21. En todos los demás objetos educativos enriquecidos con RA no encontramos diferencias llamativas en las valoraciones en relación al género.

Como respuesta a la tercera pregunta de investigación (¿Los estudios de procedencia del alumnado influyen de forma significativa a la hora de valorar objetos educativos enriquecidos con RA?) se puede señalar que el área de conocimiento a la que pertenecen los estudiantes que experimentaron con RA en esta investigación no influye de forma significativa en sus valoraciones. Es decir, no se han encontrado diferencias significativas entre los grupos implicados en función de la rama de conocimiento de los estudiantes: Arte y Humanidades, Ciencias, Ciencias Sociales y Jurídicas e Ingeniería y Arquitectura.

A pesar de no existir estas diferencias significativas se puede señalar que los que mejor valoraron los objetos fueron los alumnos pertenecientes al área de Arte y Humanidades, mientras que los que peor los valoran son los de Ciencias e Ingeniería y Arquitectura.

Un análisis diferenciador nos confirma que son los alumnos de Arte y Humanidades quienes obtienen la media más alta en los tres bloques de características en cinco objetos, y no destacan por valorar negativamente ningún apartado de ninguno de los objetos educativos enriquecidos con Realidad
Aumentada. Los egresados de la rama de Ciencias obtienen la mejor media en valoraciones positivas solamente en el factor Guía/Tutorial del programa del objeto 9 o Formas de utilizar el vídeo en la enseñanza $(3,33)$. Dos veces los alumnos de Ingeniería y Arquitectura, en los factores Aspectos técnicos y estéticos y Guía/Tutorial del programa en el objeto 5, 10,00 y 5,60 respectivamente. $Y$ tres veces los estudiantes de Ciencias Sociales y Jurídicas en los factores Facilidad de navegación y desplazamiento por el entorno, y Guía/Tutorial del programa en el objeto 7, 9,80 y 3,14 respectivamente, y en Aspectos técnicos y estéticos del objeto número 8 o Diseño, producción y evaluación de Tecnologías de la Información y Comunicación aplicadas a la educación, con una media de $(6,67)$. En todos los casos estas medias están muy cerca de la media positiva obtenida por el alumnado de Arte y Humanidades.

En cambio, los que vierten valoraciones más negativas son los de Ciencias, que evalúan alguno o todos los aspectos de forma negativa en ocho objetos (valoración negativa total en los objetos 1,3 , y valoración negativa parcial en los objetos 2, 4, 5, 7, 8 y 9). Esto es, el único objeto donde no obtienen la mayor media en valoración negativa es el objeto número 6 o Anatomía humana: tobillo, que es peor valorado por Ingeniería y Arquitectura y Ciencias Sociales y Jurídicas.

En la misma línea se encuentran los alumnos de Ingeniería y Arquitectura, que consiguen la mayor media en relación a la valoración negativa que hacen de cinco objetos.

También debemos destacar que la diferencia entre medias de las valoraciones positivas y negativas es inferior a 5 puntos en todos los casos, menos en dos: en el apartado Facilidad de navegación y desplazamiento por el entorno del objeto $4 \mathrm{o}$

$-28-$ 
Anatomía humana: hombro $(5,3)$, y en el mismo apartado del objeto 2 o Anatomía humana: coxofemoral (5). Además en tres ocasiones la diferencia es inferior a uno: En el apartado de Guía/Tutorial del programa en el objeto número $7(0,74)$ y en el mismo apartado del objeto 8 o Diseño, producción y evaluación de Tecnologías de la Información y Comunicación aplicadas a la educación $(0,92)$ y del objeto 4 o Anatomía humana: hombro $(0,84)$. En este último objeto llama la atención que coinciden en la media de la valoración negativa alumnos de dos áreas de conocimiento, los de Ciencias Sociales y Jurídicas y los de Ingeniería y Arquitectura. Hecho que solamente se repite una vez más, en el factor Guía/Tutorial del programa del objeto número dos, donde obtienen el mismo resultado referente a la valoración negativa (2.00) los estudiantes de Ciencias y los de Ingeniería y Arquitectura.

\section{Conclusiones.}

Los objetos de aprendizaje enriquecidos con Realidad Aumentada obtienen una valoración positiva, destacando entre el ellos el objeto 3, Histología humana: hígado. Coincide que éste es el primer objeto con una figura sobreimpresionada que se presentaba a los alumnos para su valoración. Este factor favorece que el lanzador sea muy rápido y el objeto RA que emerge salga de inmediato, hecho que no ocurre con la misma velocidad en los dos objetos anteriores. Por ello parece lógico pensar que esta característica técnica pueda ser la causa de su valoración positiva, ya que otros objetos de estas características también han sido valorados positivamente, entre ellos el objeto 5 o Histología humana: pulmón.

En cambio en el objeto 1, Anatomía humana: cervical, tal y como ocurre en otros objetos como el 2 (Anatomía humana: coxofemoral) o el 6 (Anatomía humana: tobillo) el lanzador no es tan rápido, lo que ralentiza un poco el proceso. Este objeto 1, Anatomía humana: cervical, es el único que presenta diferencias significativas de valoración entre los grupos de edad. Recordemos que los estudiantes entre 20 y 29 lo valoraban de forma mucho más positiva que los mayores de 30. Estos resultados se pueden ver justificados porque, a pesar de no ser un lanzador tan rápido, el efecto sorpresa influye en mayor medida entre el alumnado más joven y pesa más que la facilidad técnica al comienzo de la experiencia educativa de valoración de los objetos enriquecidos con RA.

Otra razón está unida a la propia competencia digital, e incluso a la cercanía de uso. No cabe duda de que el uso de objetos educativos enriquecidos con Realidad Aumentada exige un nivel de competencia digital alto en los usuarios. Pero además de la competencia parece también interesante y necesario indagar sobre otro factor: la familiaridad de uso. Esto es, el haber utilizado anteriormente la RA puede conllevar una mejor valoración del propio producto.

Este último es un aspecto que se consolida cuando hablamos de los estudios que han cursado los estudiantes de máster con anterioridad, ya que hemos visto que los que mejor valoran los objetos son los que provienen de las Artes y Humanidades. Este dato corrobora el aportado en su estudio por Bacca, Baldiris, Fabregat, Graf y Kinshuk (2014) donde se señalaba que las ramas de conocimiento donde más se había experimentado con Realidad Aumentada era en Arte y Humanidades. Por lo que, tal y como hemos señalado sería interesante seguir investigando esta variable de familiaridad de uso unida a la satisfacción hacia su uso.

-29 -

Píxel-Bit. Revista de Medios y Educación. No 50 Enero 2017. ISSN: 1133-8482. PIXEL e-ISSN: 2171-7966. doi: http://dx.doi.org/10.12795/pixelbit.2017.i50.01 
Otro dato que destaca es que la figura peor valorada, la número 7, Mapa interactivo de los Jardines del Monasterio de Cartuja de Sevilla, es una de las más completas y novedosas, pero que exige tener la geolocalización activada en el dispositivo móvil. Se puede considerar por tanto que puede tener mayores dificultades técnicas y que eso revierte en la dificultad de comprender su uso y su valoración negativa por parte del alumnado participante en la experiencia. Aspecto que viene reforzado en que entre las tres características susceptibles de apoyar la mejora en el aprendizaje ( 1. Aspectos técnicos y estéticos; 2. Facilidad de navegación y desplazamiento por el entorno y 3. Guía/Tutorial del programa) la mejor valorada haya sido la Facilidad de navegación y desplazamiento por el objeto.

El alumno parece valorar que el objeto sea lo más intuitivo y rápido posible y con un funcionamiento técnico fácil, donde haya diferentes materiales como objetos 3D manipulables y vídeos de reducido tamaño. En esta línea sería interesante profundizar en la valoración que se hace de estos materiales que emergen de los lanzadores. Además, y a tenor de los resultados donde no se encuentran diferencias significativas ni en las características intrínsecas ni extrínsecas de los estudiantes en cuanto a su valoración, podría pensarse en buscar otras variables como la motivación, el rendimiento académico o las propias emociones con el uso de la Realidad Aumentada en materiales educativos en la universidad.

\section{Fuentes de financiación.}

El trabajo se enmarca dentro de un proyecto de investigación $\mathrm{I}+\mathrm{D}$ financiado por el Ministerio de Economía y Competitividad del Gobierno de España denominado: "Realidad aumentada para aumentar la formación. Diseño, producción y evaluación de programas de realidad aumentada para la formación universitaria" (EDU-5746-PProyecto Rafodiun).

\section{Referencias bibliográficas.}

Akçayir, M., Akçayir, G., Pektas, H.M. \& Ocak,M.A. (2016). Augmented reality in science laboratories: The effects of augmented reality on university students'laboratory skills and attitudes toward science laboratories. Computers in Human Behavior, 57, 334-342. doi:10.1016/ j.chb.2015.12.054

Bacca, J., Baldiris, S., Fabregat, R., Graf, S. \& Kinshuk (2014). Augmented Reality Trends in Education: A Systematic Review of Fesearch and Apllications. Educational Technology \& Societi, 17 (4), 133-149. Recuperado de http://www.ifets.info/journals/ 17_4/9.pdf(28/05/2016).

Basogain, X., Olabe, M., Espinosa, K., Roueche, C. \& Olabe, J.C. (2007). Realidad Aumentada en la Educación: una tecnología emergente. Recuperado de http:// www.anobium.es/docs/gc_fichas/doc/ 6CFJNSalrt.pdf(14/05/2016).

Bongiovani, P. (2013). Realidad aumentada en la escuela: Tecnología, experiencias e ideas.Educ@conTIC. Recuperado de http:// www.educacontic.es/blog/realidadaumentada-en-la-escuela-tecnologiasexperiencias-e-ideas (27/06/2016)

Cabero, J. \& Barroso, J. (2015). Realidad Aumentada: Posibilidades Educativas. En J. Ruiz, J. Sánchez \& E. Sánchez (Eds.). Innovaciones con tecnologías emergentes. (pp.1-15) Málaga: Universidad de Málaga.

Cabero, J. \& García, F. (2016). Realidad Aumentada. Tecnología para la formación. Madrid: Síntesis.

-30 - 
Cabero, J., García, F. \& Barroso, J. (2016). La producción de objetos de aprendizaje en «Realidad Aumentada»: la experiencia del SAV de la Universidad de Sevilla. International Journal of Educational Research and Innovation (IJERI), 6, 110-123.

Castaño, C. \& Cabero, J. (2013). Enseñary aprender en entornos m-learning. Madrid: Síntesis.

Chiu, J.L., DeJaegher, C.J. \& Chao, J. (2015). The effects of augmented virtual science laboratories on middle school-students' understanding of gas properties. Computers \& Education, 85, 59-73. doi:10.1016/ j.compedu.2015.02.007

De Pedro, J. \& Méndez, C.L.M. (2012). Realidad aumentada una alternativa metodológica en Educación Primaria nicaragüense. IEES-RITA, 7, 102-108.

Durall, E., Gros, B., Maina, M., Johnson, L. \& Adams, S. (2012). Perspectivas tecnológicas: educación superior en Iberoamérica 2012-2017. Austin, Texas: The New Media Consortium. Recuperado de http://www.nmc.org/pdf/2012-technologyoutlook-iberoamerica_SP.pdf(15/05/2016).

ElSayed, N.A., Zayed, H.H. \& Sharawy, M.I. (2011). ARSC: augmented reality student card. Computers \& Education, 56, 1045-1061. doi:10.1016/j.compedu.2010.10.019

García, I., Peña, I., Johnson, L., Smith, R., Levine, A. \& Haywood, K (2010). Informe Horizon: Edición Iberoamericana 2010. Austin Texas: The New Media Consortium. Recuperado de http://www.nmc.org/pdf/2010Horizon-Report-ib.pdf(10/05/2016).

Gutierrez, I. (2014). Perfil del profesor universitario español en torno a las competencias en tecnologías de la información y la comunicación. Pixel Bit. Revista de Medios y Educación, 44, 51-65. doi: http://dx.doi.org/10.12795/ pixelbit.2014.i44.04
Johnson, L., Adams, S., Cummins, M., Estrada, V., Freeman, A. \& Ludgate, H. (2013). Techology Outlook for Australian Tertiary Education 2013-2018: An NMC Horizon Project Regional Analysis. Austin, Texas: The New Media Consortium. Recuperado de http://www.nmc.org/pdf/2013-TechnologyOutlook-for-Australian-TertiaryEducation.pdf(12/05/2016).

Muñoz, J.M. (2013). Realidad Aumentada, realidad disruptiva en las aulas. Boletín SCOPEO, 82. Recuperado de http:// scopeo.usal.es/realidad-aumentada-realidaddisruptiva-en-las-aulas/ (12/05/2016).

Pei, E.L. \& Ming, T. (2013). Using augmented-reality-based mobile leraning material in EFL English compositivon: An exploratory case study. British Journal Educational Technology, 4 (1). Recuperado de http://onlinelibrary.wiley.com/doi/10.1111/ j.1467-8535.2012.01302.x/abstract (28/06/ 2016). doi: 10.1111/j.1467-8535.2012.01302.x

Prendes, C. (2015). Realidad Aumentada y Educación. Análisis de experiencias prácticas. Pixel-Bit. Revista de Medios y Educación, 46, 187-203. doi: http://dx.doi.org/10.12795/ pixelbit.2015.i46.12.

UNESCO (2012). Turning on mobile learning. Global Themes. París: Unesco.

Wu, H-S., Wen, S. \& Yi, H. (2013). Current status, opportunities and challenges of augmented reality in education. Computers \& Education, 62, 41-49. doi: 10.1016/ j.compedu.2012.10.024. doi: 10.1016/ j.comopedu.2012.10.024

Fecha de recepción: 10-06-2016

Fecha de evaluación: 13-06-2016

Fecha de aceptación: 12-09-2016 $-31-$

Píxel-Bit. Revista de Medios y Educación. $N^{o} 50$ Enero 2017. ISSN: 1133-8482. e-ISSN: 2171-7966. doi: http://dx.doi.org/10.12795/pixelbit.2017.i50.01 